\title{
99m Tc-CXCL8 SPECT to Monitor Disease Activity in Inflammatory Bowel Disease
}

\author{
Erik H.J.G. Aarntzen ${ }^{1}$, Rick Hermsen ${ }^{1,2}$, Joost P.H. Drenth ${ }^{3}$, Otto C. Boerman ${ }^{1}$, \\ and Wim J.G. Oyen ${ }^{1}$ \\ ${ }^{1}$ Department of Radiology and Nuclear Medicine, Radboudumc, Nijmegen, The Netherlands; ${ }^{2}$ Department of Nuclear Medicine, \\ Canisius Wilhelmina Ziekenhuis, Nijmegen, The Netherlands; and ${ }^{3}$ Department of Gastro-Enterology, Radboudumc, Nijmegen, \\ The Netherlands
}

Inflammatory bowel diseases (IBDs) are defined as chronic relapsing immune-mediated disorders of the gastrointestinal tract. IBD exacerbations are characterized by recruitment of mainly CXCL8 receptorexpressing activated neutrophils into the intestinal wall, leading to severe damage. Considering its chronic relapsing character, accurate and timely diagnosis of an exacerbation is pivotal for early adaptation of the treatment and reduction of the disease burden. However, endoscopic evaluation is invasive and associated with an increased risk of perforation. We previously developed a ${ }^{99 m}$ Tc-labeled CXCL8 preparation in preclinical models including colitis and clinical studies. Methods: In this study, we investigate the accuracy of ${ }^{99 m T c-C X C L 8}$ SPECT to detect and localize disease activity in a prospective series of patients with IBD. Thirty patients (15 Crohns disease, 15 ulcerative colitis) participated, and 92 segmental pairs of histology and $99 \mathrm{mTc}-$ CXCL8 scans were studied. Imaging was performed after injection of $400 \mathrm{MBq}$ of ${ }^{99 m T C}-C X C L 8$. Planar and SPECT images of the abdomen were acquired at $30 \mathrm{~min}$ and $4 \mathrm{~h}$ after the injection. Results: The overall sensitivity and specificity on a per-patient basis for the detection of active disease were $95 \%$ and $44 \%$ for ${ }^{99 m T c-C X C L 8 ~ s c a n ~}$ and $71 \%$ and $70 \%$ for endoscopy. The degree of ${ }^{99 m T c-C X C L 8 ~ a c-~}$ cumulation correlated to the degree of neutrophilic influx in affected mucosa. Sensitivity and specificity on a per-segment basis, calculated from the 92 segmental pairs, were $82 \%$ and $72 \%$, negative predictive value was $81 \%$, and overall positive predictive value was $74 \%$. Specificity could be increased at the expense of sensitivity using different cutoffs. In 74 segmental pairs, overall sensitivity and specificity for endoscopy were $74 \%$ and $85 \%$, positive predictive value was $81 \%$, and negative predictive value was $79 \%$. Conclusion: 99mTc-CXCL8 SPECT provides a novel imaging technique to target neutrophil recruitment to the intestinal wall, especially in moderate to severe exacerbations of IBD. Further validation studies are warranted to potentiate ${ }^{99 m T C-C X C L 8 ~ S P E C T ~ a s ~ a ~ b i o m a r k e r ~ t o ~ s c a l e ~ u p ~ o r ~ s t e p ~}$ down treatment with immune-modulating drugs in a personalized fashion.

Key Words: CXCL8; inflammatory bowel disease; neutrophil recruitment; scintigraphy; SPECT

J Nucl Med 2016; 57:398-403

DOI: 10.2967/jnumed.115.165795

Received Aug. 23, 2015; revision accepted Nov. 10, 2015.

For correspondence or reprints contact: Erik H.J.G. Aarntzen, Radboudumc, Department of Radiology and Nuclear Medicine, P.O. Box 9101, 6500HB,

Nijmegen, The Netherlands.

E-mail: erik.aarntzen@radboudumc.nl

Published online Nov. 25, 2015.

COPYRIGHT (C) 2016 by the Society of Nuclear Medicine and Molecular Imaging, Inc.
$\mathbf{I}$ lapsing immune-mediated disorders of the gastrointestinal tract. Although the antigenic trigger remains elusive, IBD exacerbations are characterized by an inappropriate and extensive recruitment of immune cells into the intestinal wall (1). Those activated immune cells, predominantly neutrophils, CD8 $+\mathrm{T}$ cells, and macrophages, release proinflammatory cytokines, eventually leading to mucosal damage. The extent of inflammation differs with type of IBD and the abnormal inflammatory response is typically transmural, with deep ulcers, fissures, and fibrosis in Crohn disease and restricted to the mucosa in ulcerative colitis. Current treatment options with local or systemic steroids and biologicals aim at curtailing the immune response.

The chronic relapsing character of IBD not only is the central issue in disease management, it also results in high direct and indirect costs with a considerable economic burden on health care systems (2). Moreover, IBD is associated with mucosal damage and extensive fibrosis, giving rise to a wide spectrum of diseaseassociated complications such as iron-deficient anemia, bowel stenosis, and colorectal carcinoma eventually requiring surgical resection (3). Accurate and timely diagnosis of an exacerbation is pivotal to early adaptation of the treatment and reduction of the disease burden.

Central to the diagnostic procedures in IBD is endoscopy, which allows histologic confirmation of the affected lesions. However, endoscopy is invasive and requires bowel preparation, which is poorly accepted by patients (4). Moreover, severe colitis is considered as a relative contraindication for complete colonoscopy because of an increased risk of perforation $(5,6)$. Several noninvasive imaging techniques have been developed to diagnose exacerbations and assess the number and severity of IBD lesions in patients. Currently, CT and occasionally ${ }^{18}$ F-FDG PET/CT are used clinically, although other scintigraphic techniques have been tested $(7,8)$. Detection of IBD lesions by CT relies on the morphologic changes of the gut wall caused by severe local inflammation (edema, thickening) (9). The sensitivity of CT for detection of lesions, endoscopically characterized as large superficial ulcers, is $56 \%$ and increases to $86 \%$ for deep ulcers and strictures. ${ }^{18} \mathrm{~F}-\mathrm{FDG}$ PET detection is based on increased metabolic activity of the infiltrated immune cells and accessory cells in the inflamed gut wall. Overall sensitivity for ${ }^{18} \mathrm{~F}$-FDG PET is $85 \%$ (10), varying from $50 \%$ for nonulcerated lesions to $100 \%$ for deep ulcers (9). Although specificity during exacerbations is reasonable, $87 \%$ (95\% confidence interval [CI], 84\%-90\%) (10), highly variable physiologic 
${ }^{18} \mathrm{~F}-\mathrm{FDG}$ uptake in the colon limits its use in screening and followup. Both imaging modalities correctly localize disease $(11,12)$; contrast-enhanced CT scans require substantial changes to the gut wall for sensitive detection and ${ }^{18}$ F-FDG PET requires severe infiltration of metabolically active immune cells before its uptake exceeds physiologic uptake and therefore both modalities fail to detect IBD lesions in their early phase.

Radiolabeled CXCL8 (interleukin-8) is an interesting candidate because it targets the CXCL8 receptors (CXCR1 and CXCR2) mediating chemotaxis of immune cells to the site of inflammation (13). Neutrophils, and to a lesser extent CD8 + T cells and macrophages, express both CXCR1 and CXCR2 (14-16). Mouse models and clinical studies have demonstrated a direct correlation between the degree of histologic inflammation and CXCL8 concentration in IBD (17). The expression of CXCR1 and CXCR2 is increased in inflamed IBD tissue on an RNA and protein level (18). Moreover, triggering CXCR1 has a role beyond immune cell recruitment, because it unleashes the effector functions responsible for the potential damage to the gut wall (19).

We developed a ${ }^{99 \mathrm{~m} T c-l a b e l e d ~ C X C L 8}$ preparation that has excellent characteristics for imaging infection and inflammation in various preclinical models including colitis (20) and in patients suspected of focal infections (21). In the present study, we explored the diagnostic accuracy of ${ }^{99 \mathrm{~m} T c-C X C L 8}$ scintigraphy to detect and localize disease activity in patients with IBD prospectively.

\section{MATERIALS AND METHODS}

\section{Study Population}

Patients who were scheduled for a conventional colonoscopy to assess disease activity or routine follow-up or when they were admitted because of active colitis were eligible for the study. Pregnancy and inability to lie supine for more than $30 \mathrm{~min}$ were exclusion criteria. The study was approved by the regional ethical review board (CMO Arnhem-Nijmegen). All subjects gave their written informed consent.

\section{9mTc-CXCL8 Preparation}

${ }^{99 m}$ Tc-CXCL8 was produced as described before (21). Synthetic human CXCL8 was synthesized under conditions of good manufacturing practice by RMF Dictagene SA. Lyophilized samples of $250 \mu \mathrm{g}$ were stored at $4{ }^{\circ} \mathrm{C} . N$-[tris(hydroxymethyl)-methyl]glycine (Tricine) was purchased from Fluka. Glycine, nicotinic acid, polyoxyethylenesorbitan monooleate (polysorbate 80 ), and 2-( $N$-morpholino)ethanesulfonic acid were purchased from Sigma-Aldrich. The propylaldehyde hydrazone of succinimidyl-hydrazinonicotinic acid was synthesized as described previously (22).

The conjugation of CXCL8 to 6-hydrazinopyridine-3-carboxylic acid (HYNIC) was performed as follows: a sample of $250 \mu \mathrm{g}$ of synthetic human CXCL8 was reconstituted in $45 \mu \mathrm{L}$ of buffer $(50 \mathrm{mM} 2-(\mathrm{N}-$ morpholino)ethanesulfonic acid, $\mathrm{pH} 6.5$, and $0.32 \mathrm{M} \mathrm{NaCl})$, and $5 \mu \mathrm{L}$ of $1.0 \mathrm{M} \mathrm{NaHCO}_{3}, \mathrm{pH} 8.2$, were added. Subsequently, a 3-fold molar excess of HYNIC in $5 \mu \mathrm{L}$ of dry dimethyl sulfoxide was added to the mixture. After incubation for $10 \mathrm{~min}$ at room temperature, the reaction was stopped by the addition of an excess of glycine $(50 \mu \mathrm{L} 1.0 \mathrm{M}$ glycine in phosphate-buffered saline). Next, $0.9 \mathrm{~mL}$ of phosphatebuffered saline was added, and the mixture was extensively dialyzed against phosphate-buffered saline in a 0.5 - to $3.0-\mathrm{mL}$ Slide-A-Lyzer with a molecular weight cutoff of 3,500 (Pierce) to remove excess unbound HYNIC. After dialysis, polysorbate 80 to a final concentration of $0.1 \%$ was added. Samples of $10 \mu \mathrm{g}$ of CXCL8-HYNIC in $400 \mu \mathrm{L}$ were stored at $-20^{\circ} \mathrm{C}$

For radiolabeling, a lyophilized coligand kit was prepared containing $30 \mu \mathrm{g}$ of $\mathrm{SnSO}_{4}, 45 \mathrm{mg}$ of $N$-[tris(hydroxymethyl)-methyl]glycine, and
$4 \mathrm{mg}$ of nicotinic acid. The coligand kit was reconstituted with $0.6 \mathrm{~mL}$ of a $0.9 \% \mathrm{NaCl}$ solution immediately before the start of the radiolabeling procedure. To a $10-\mu \mathrm{g}$ CXCL8-HYNIC sample, $400 \mu \mathrm{L}$ of a reconstituted coligand kit were added, together with $800-1,000 \mathrm{MBq}$ of ${ }^{99} \mathrm{mc}^{\mathrm{T}} \mathrm{O}_{4}{ }^{-}$in saline. The mixture was incubated at $70^{\circ} \mathrm{C}$ for $30 \mathrm{~min}$. The radiochemical purity was determined by instant thin-layer chromatography (ITLC) on ITLC-silica gel strips with $0.1 \mathrm{M}$ citrate, $\mathrm{pH} 6.0$, as the mobile phase. After the labeling reaction, the reaction mixture was diluted in $0.9 \% \mathrm{NaCl}$ to a final concentration of $40 \mathrm{MBq} / \mathrm{mL}$. The radiochemical purity of all preparations exceeded $90 \%$. Ten milliliters of the final preparation was intravenously administered to a patient over 5 min using an infuser (Asena; Alaris Medical Systems).

\section{Image Acquisition}

Imaging was performed after injection of $400 \mathrm{MBq}$ of ${ }^{99 \mathrm{~m}} \mathrm{Tc}-$ CXCL8 (protein dose, $5 \mu \mathrm{g}$ ). The scintigraphic protocol included planar and tomographic (SPECT) acquisitions of the abdomen. All images were acquired using a dual-head, $\gamma$-camera (ECAM; Siemens Inc.) equipped with 2 low-energy, high-resolution collimators. Planar images (anterior and posterior abdominal-pelvic projections) were acquired at $30 \mathrm{~min}$ and $4 \mathrm{~h}$ after the injection of ${ }^{99 \mathrm{~m} T \mathrm{~T}-\text { CXCL8. }}$ A SPECT study was performed just after the completion of the 4-h postinjection planar images. SPECT was acquired using a matrix size of $128 \times 128,360^{\circ}$ circular rotation, and a $3^{\circ}$ angle step with a 20 - to 25 -s time frame.

\section{Image Analysis}

A predefined abdominal map with regions for ascending colon, transversal colon, descending colon, and terminal ileum was used for scoring. Standard regions of interest were drawn on the anterior images acquired at $4 \mathrm{~h}$ after injection over the liver (avoiding kidney and costochondral junctions), spleen (avoiding kidney), and left greater trochanter and soft-tissue lateral to the greater trochanter (Supplemental Fig. 1; supplemental materials are available at http:// jnm.snmjournals.org). Regions of interest were mirrored on the posterior images, and geometric mean was taken to calculate activity (counts/s). ${ }^{99 \mathrm{~m} T c-C X C L 8}$ uptake of each colonic segment was graded on a $0-3$ scale, for both images acquired in anteroposterior view and by emission (SPECT) acquisitions (0, absent uptake; 1 , faint uptake less than bone marrow; 2, uptake equal to bone marrow; 3 , uptake more than bone marrow) on consecutive transverse sections including the entire bowel uptake volume. As compared with spleen, liver, or soft-tissue activity, bone marrow activity was the most reproducible and constant (Supplemental Fig. 1); therefore, we considered bone marrow activity to be the best internal reference. A global score of 0 as assessed by either anteroposterior or SPECT images defined a negative scintigraphy. Scintigraphic images were scored independently by 2 nuclear medicine physicians masked for patients' clinical details. The scans with equivocal findings were resolved by consensus, with a third observer as referee.

\section{Endoscopic Evaluation}

Flexible endoscopy procedures were performed after thorough oral lavage using macrogol 3350 (Klean-Prep, various manufacturers). The procedure was performed by a fellow-endoscopist, directly supervised by an experienced gastroenterologist, using a conventional colonoscope (Olympus CF 140 or PCF160; Olympus Optical). We used common endoscopic landmarks to distinguish 4 different segments (ascending colon, transversal colon, descending colon, and terminal ileum). The endoscopic findings of inflammatory activity were classified as absent (grade 0, no inflamed mucosa), mild (grade 1, granularity, edema, invisible vascular pattern), moderate (grade 2, hyperemia, friability, plus all features of mild inflammatory activity), or severe (grade 3, ulceration in addition to features of moderate inflammatory activity). A positive endoscopy was scored when there were at least signs of mild inflammatory 
activity. In the case colonoscopy was contraindicated in patients with severely active colitis, we judged the extent of inflammatory changes in these patients using abdominal CT scanning or by macroscopic examination of the surgically excised colon.

\section{Histologic Evaluation}

Histopathologic inflammation was graded as absent (grade 0), mild (grade 1), moderate (grade 2), or severe (grade 3). Mild inflammation reflected a slight increase in mononuclear cells within the lamina propria. Moderate inflammation described a marked increase of mononuclear cells within the lamina propria and foci of cryptitis but with relatively preserved glandular architecture. Severe inflammation described extensive glandular damage and extensive neutrophil infiltration of the epithelium along with a marked increase in mononuclear cells within the lamina propria.

\section{Statistical Analysis}

To compare the results between groups of patients, a 2-tailed MannWhitney $U$ test was used. Sensitivity, specificity, positive predictive value (PPV), and negative predictive value (NPV) were assessed by the Fisher exact 2-tailed $t$ test, and the correlation coefficient (r) was determined using the student paired $t$ test. $P$ values of less than 0.05 were considered significant. Analyses were performed using Prism software (version 5.0; GraphPad).

\section{RESULTS}

\section{Patient Characteristics}

Thirty-one scans were acquired for 30 patients ( 8 men, 22 women); 1 patient was scanned during clinical exacerbation and during follow-up (Table 1). The mean age was 41.3 y (range, 18.8$66.8 \mathrm{y}$ ). The age at the onset of disease was $30.9 \mathrm{y}$ (range, 15-65 y).

\section{TABLE 1}

Study Population Characteristics

( $n=30$ Patients, $n=31$ Scans)

\begin{tabular}{|ll}
\hline \multicolumn{1}{c}{ Characteristic } & \multicolumn{1}{c}{$n$} \\
\hline Sex & Men, 8 \\
\hline & Women, 22 \\
\hline IBD type & Ulcerative colitis, 15 \\
\hline Referral type & Crohn disease, 15 \\
\hline Time to histology (d) & Follow-up, 14 \\
\hline Time to endoscopy (d) & Median, $2(-46$ to +92$)$ \\
\hline Therapy during scan & Median, $-2(-46$ to +87$)$ \\
\hline Systemic steroids & \\
\hline Thiopurine & Yes, 19 \\
\hline 5-ASA & No, 11 \\
\hline Local therapy & Yes, 16 \\
\hline & No, 14 \\
\hline & Yes, 15 \\
\hline & No, 15 \\
\hline
\end{tabular}

Data in parentheses are ranges. Mean age was 41.3 y (age range, 18.8-66.8 y), and mean age at onset was $30.9 \mathrm{y}$ (age range, 15-65 y).

\section{Safety}

Intravenous injection of ${ }^{99 \mathrm{~m}} \mathrm{Tc}-\mathrm{CXCL} 8$ was well tolerated. Initially, a few patients experienced mild self-limiting flushing and a mild feeling of discomfort in the upper abdomen when ${ }^{99 \mathrm{~m}} \mathrm{Tc}-$ CXCL8 was injected as a bolus injection. This disappeared quickly after injection. We observed no other significant side effects. When ${ }^{99 \mathrm{~m} T c-C X C L 8}$ was infused over a 5-min period, flushing was not observed.

\section{9mTc-CXCL8 Uptake in Intestinal Lesions Is Increased During Exacerbation}

Fifteen patients had Crohn disease and 15 patients ulcerative colitis; 17 patients were enrolled during an episode of exacerbation, and 14 patients were scanned during routine follow-up (without signs or symptoms of active disease) (Table 1). ${ }^{99 \mathrm{~m}} \mathrm{Tc}-$ CXCL8 uptake in bone marrow, spleen, liver, or soft tissue was similar in patients with or without clinical disease activity (Fig. 1). Uptake of ${ }^{99 \mathrm{~m} T c-C X C L 8}$ in intestinal lesions was significantly increased during exacerbation, also when normalized to bone marrow uptake (Fig. 1).

\section{Per-Patient Accuracy of ${ }^{99 \mathrm{~m} T c-C X C L 8}$ Imaging}

Histologic confirmation of disease activity was considered the gold standard to compare the diagnostic accuracy of ${ }^{99 \mathrm{~m}} \mathrm{Tc}$ CXCL8 scintigraphy and endoscopy on a per-patient basis. The sensitivity and specificity for $99 \mathrm{mTc}-$ CXCL8 scintigraphy were 95\% (95\% CI, 77\%-99\%) and 44\% (95\% CI, 14\%-79\%), respectively. The PPV and NPV were $81 \%$ (95\% CI, 61\%-93\%) and $80 \%$ (95\% CI, 28\%-99\%), respectively (Table 2 ).

The sensitivity and specificity of endoscopy for the detection of one or more active colitis lesions in a given patient were $71 \%$ (95\% CI, 48\%-89\%) and 70\% (95\% CI, 38\%-93\%), respectively. The PPV and NPV were $83 \%$ (95\% CI, 59\%-96\%) and 54\% (95\% CI, $25 \%-81 \%$ ).

\section{Per-Segment Accuracy of ${ }^{99 m}$ Tc-CXCL8 Imaging}

For ${ }^{99 m}$ Tc-CXCL8 SPECT, 151 segments were evaluable and scored; for endoscopy and histology, 100 and 92 segments were evaluable, respectively (Fig. 2). The main reasons for not evaluating segments by endoscopy were severe colitis, pain/discomfort, and the inability to inspect all segments. Furthermore on ${ }^{99 \mathrm{~m}} \mathrm{Tc}-$ CXCL8 SPECT, in 3 patients with Crohn disease involvement of the small intestine was noted and in 3 patients with Crohn disease involvement of the stomach. One patient had 99mTc-CXCL8 uptake in the colostomy, and 1 patient had uptake in a pelvic abscess (Supplemental Fig. 2).

Ninety-two matched segmental pairs from ${ }^{99 \mathrm{~m} T c-C X C L 8}$ scanninghistology were available (Supplemental Table 1) for analysis and 74 matched pairs from endoscopy-histology. The sensitivity and specificity for ${ }^{99 \mathrm{~m}} \mathrm{Tc}-\mathrm{CXCL8}$ scintigraphy were $82 \%$ (95\% CI, $68 \%-92 \%)$ and $72 \%$ (95\% CI, 57\%-84\%), respectively. The PPV and NPV were $74 \%$ (95\% CI, 60\%-85\%) and $81 \%$ (95\% CI, 66\%$91 \%$ ) (Table 2; Fig. 3). The sensitivity and specificity of endoscopy for the detection of a segment with active colitis were $74 \%(95 \%$ CI, 56\%-87\%) and $85 \%$ (95\% CI, 70\%-94\%), respectively. The PPV and NPV were $81 \%$ (95\% CI, 63\%-92\%) and 79\% (95\% CI, $64 \%-90 \%)$, respectively.

Review of the false-positive segments revealed that in 4 patients (patients 8, 16, 22, and 27), the ascending colon was scored as faint uptake less than bone marrow; in 1 patient (patient 27), the terminal ileum was scored, but no histologic or endoscopic evaluation was available; and in 1 patient (patient 12), the transverse colon was 


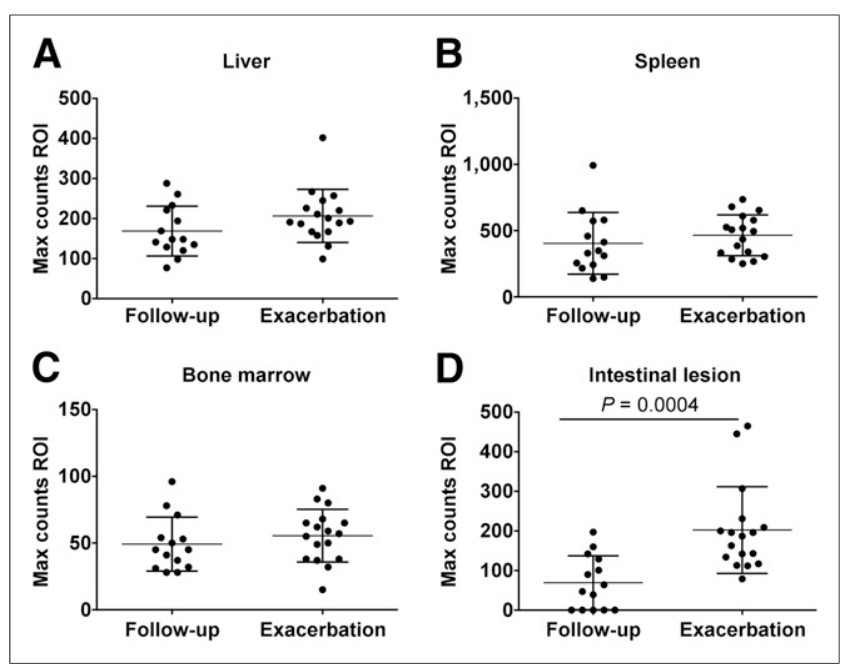

FIGURE 1. Maximum number of counts measured in regions of interest (ROIs) was comparable during exacerbation and follow-up for liver (A), spleen (B), and bone marrow (C) but was significantly higher in intestinal lesions (D) during exacerbation as compared with follow-up. Max $=$ maximum.

scored as uptake equal to bone marrow, but endoscopy showed pseudopolyps that on histology showed infiltration of lymphocytes in the lamina propria and deeper layers (Supplemental Fig. 2).

Review of the 8 false-negative segments showed that all segments were scored as grade 1 on pathology; none of the segments with grade 2 or more was falsely scored negative. One segment was located in the terminal ileum, 3 segments in the ascending colon, 2 in the transverse colon, and 2 in the descending colon.

\section{Defining Optimal Cutoff for Positivity}

To explore the optimal cutoff for defining a positive lesion, we investigated the diagnostic accuracy per segment, according to the grading on ${ }^{99 \mathrm{~m}}$ Tc-CXCL8 SPECT (Fig. 3). Compared with defining any uptake (grade 1-3) as positive, as in the per-segment analysis, defining uptake equal or more than bone marrow (grade 2-3) resulted in improved specificity and PPV, at the expense of sensitivity (Table 2; Fig. 3). Defining only uptake more than bone marrow (grade 3 ) as positive further increased the specificity and
PPV to $94 \%$ (95\% CI, 82\%-99\%) and 90\% (95\% CI, 74\%-98\%), respectively.

\section{DISCUSSION}

This study demonstrates that ${ }^{99} \mathrm{~m}$ Tc-CXCL8 uptake is increased in intestinal lesions in patients with IBD during exacerbation. 99m Tc-CXCL8 SPECT has good overall accuracy to detect active colitis in patients with IBD, with better sensitivity, PPV, and NPV than endoscopy. On a per-segment analysis, ${ }^{99 m}$ Tc-CXCL8 SPECT and endoscopy perform equally well in terms of sensitivity, but endoscopy has a better specificity, resulting in slightly better PPV and a similar NPV.

The strength of this explorative study is the enrolment of patients in presence and absence of clinical disease activity, which allows an accurate assessment of the diagnostic accuracy. We demonstrate that ${ }^{99 \mathrm{~m} T c-C X C L 8}$ uptake in the bone marrow remains constant with only little variation in uptake among patients, also during exacerbation. Therefore, bone marrow uptake can serve as a reference to grade ${ }^{99 \mathrm{~m}} \mathrm{Tc}$-CXCL8 uptake in intestinal lesions. If uptake in lesions is equal to or higher than uptake in the bone marrow, ${ }^{99} \mathrm{~m}$ Tc-CXCL8 SPECT has a fairly good diagnostic accuracy for detecting disease activity. Lesions with pathology grade 2 or 3 inflammation were in all cases scored positive, and only 8 segments with pathology grade 1 were scored false-negative. The total of these 8 segments was scored within 4 patients; 3 of these patients had more segments with higher grade inflammation that were scored correctly as positive on the SPECT, and in 1 patient the transverse colon was scored false-negative, whereas endoscopy reported remarkable scar formation and pathology reported focal mixed cellular infiltration of the lamina propria, no granulomas (patient 24). Given these findings, the high NPV warrants ${ }^{99 \mathrm{~m} T c-C X C L 8}$ SPECT as a noninvasive alternative for endoscopy to rule out clinically relevant (grade 2 or more) colitis.

Four of the 6 false-positive segments were located in the ascending colon and all were scored as grade 1 only, with a typical pattern of diffuse uptake only slightly above background. Although the number of observations in this study is too small to draw conclusions, it is tempting to speculate on a relation with the composition of the microbiome and its role in triggering exacerbations in IBD patients $(23,24)$. Furthermore, in some cases increased ${ }^{99 m}$ Tc-CXCL8 uptake was observed without corresponding features

TABLE 2

Diagnostic Accuracy of $99 \mathrm{mTC}-\mathrm{CXCL8}$ SPECT

\begin{tabular}{|c|c|c|c|c|}
\hline Analysis & Sensitivity & Specificity & PPV & NPV \\
\hline \multicolumn{5}{|l|}{ Per-patient $(n=31)$} \\
\hline 99mTc-CXCL8 SPECT & $95 \%(77 \%-99 \%)$ & $44 \%(14 \%-79 \%)$ & $81 \%(61 \%-93 \%)$ & $80 \%(28 \%-99 \%)$ \\
\hline Endoscopy & $71 \%(48 \%-89 \%)$ & $70 \%(38 \%-93 \%)$ & $83 \%(59 \%-96 \%)$ & $54 \%(25 \%-81 \%)$ \\
\hline \multicolumn{5}{|l|}{ Per-segment } \\
\hline 99mTc-CXCL8 SPECT $(n=92)$ & $82 \%(68 \%-92 \%)$ & $72 \%(57 \%-84 \%)$ & $74 \%(60 \%-85 \%)$ & $81 \%(66 \%-91 \%)$ \\
\hline Endoscopy $(n=74)$ & $74 \%(56 \%-87 \%)$ & $85 \%(70 \%-94 \%)$ & $81 \%(63 \%-92 \%)$ & $79 \%(64 \%-90 \%)$ \\
\hline \multicolumn{5}{|l|}{ Per-segment, for different cutoffs } \\
\hline$\geq$ Bone marrow (grade $2-3$ ) & $69 \%(53 \%-82 \%)$ & $85 \%(72 \%-94 \%)$ & $82 \%(66 \%-92 \%)$ & $74 \%(60 \%-85 \%)$ \\
\hline Bone marrow (grade 3) & $62 \%(47 \%-76 \%)$ & $94 \%(82 \%-99 \%)$ & $90 \%(74 \%-98 \%)$ & $72 \%(59 \%-83 \%)$ \\
\hline
\end{tabular}

Data in parentheses are ranges. 


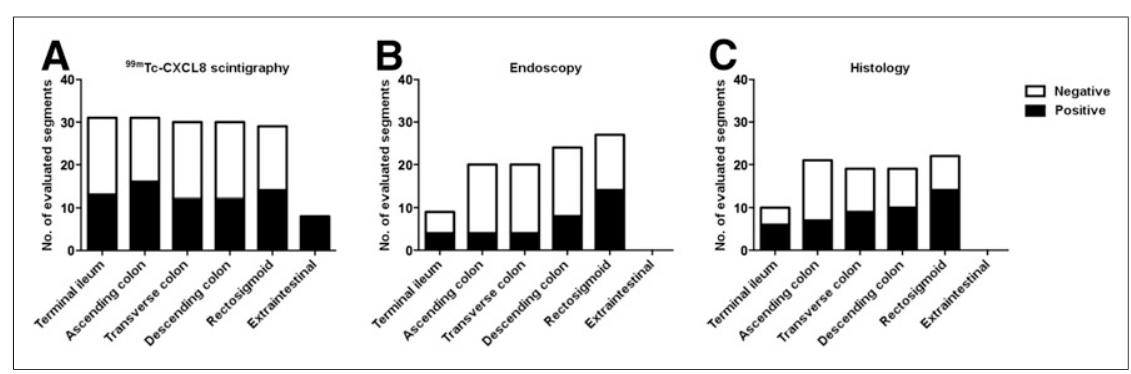

FIGURE 2. Total number of evaluated segments for ${ }^{99 m T C-C X C L 8 ~ s c i n t i g r a p h y ~(A), ~ e n d o s c o p y ~}$ (B), and histology (C) shows that more segments can be evaluated by noninvasive imaging than by endoscopic procedures, including evaluation of extraintestinal lesions or complications. No. = number.

of inflammation at endoscopy. One possible explanation is that in these patients, the colon was transmurally involved, but the center of inflammation might be deeper than the mucosa, explaining the absence of inflammation by endoscopy.

Several limitations of this explorative study should be addressed. First, the study population is heterogeneous with respect to disease entity, prior and current therapy, and type of therapy, factors that might influence the level of neutrophil recruitment to the intestinal wall. Second, the diagnostic workup was not standardized, resulting in different time spans between ${ }^{99 \mathrm{~m} T c-C X C L 8}$ scintigraphy and endoscopy/histology, and not all segments were biopsied. Especially for the distal segments, terminal ileum, and ascending colon, histologic confirmation of the ${ }^{99 \mathrm{~m}}$ Tc-CXCL8 scan results was lacking. This, together with the findings of extraintestinal involvement in patients with Crohn disease (Supplemental Fig. 2 , stomach uptake and pelvic abscess), illustrates the advantages of a noninvasive whole-body imaging procedure.

Most colonic segments were scored equivocally, because 99m Tc-CXCL-8 scintigraphy has almost no physiologic background in these regions. However, correct scoring of the transverse colon was hampered by overlay of kidneys, spleen, liver, and spine, es-

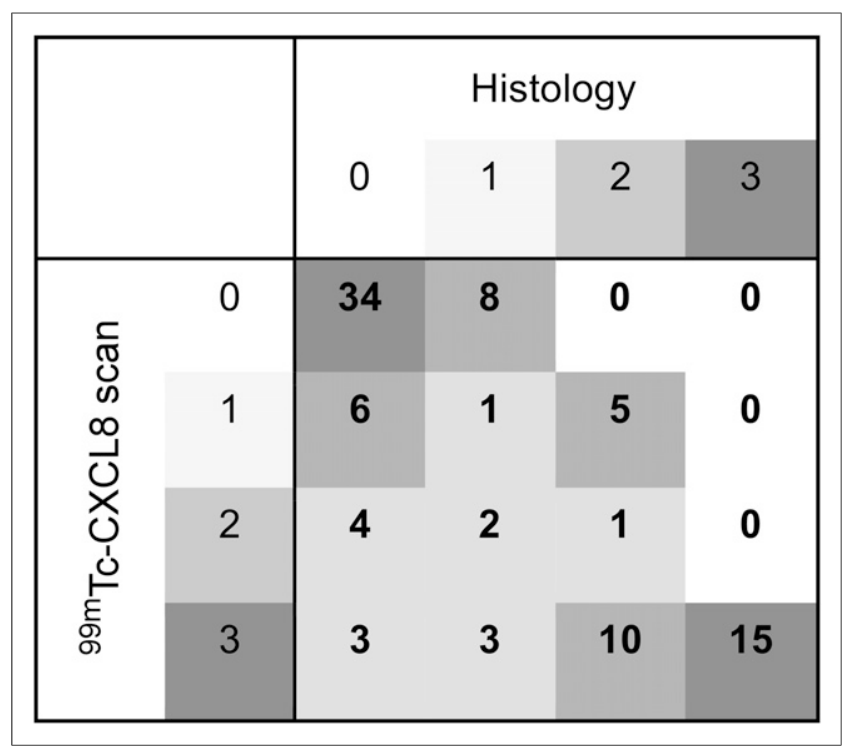

FIGURE 3. Grade of ${ }^{99 m T C-C X C L 8}$ uptake per segment correlates with grade of inflammation as evaluated by histology. Different gray tones indicate relative frequencies of correlation. pecially in the planar scans (Supplemental Fig. 3). Moreover, in some patients, endoscopy and ${ }^{99 m}$ Tc-CXCL8 scintigraphy localized IBD lesions differently. Both issues can readily be improved using SPECT/CT, which will allow the 3-dimensional localization of IBD lesions in their anatomic context, especially in cases of altered anatomy due to prior surgery.

The diagnostic performance of ${ }^{18} \mathrm{~F}-\mathrm{FDG}$ PET in IBD was reviewed by Treglia et al., concluding on a per-segment analysis that the overall sensitivity and specificity were $85 \%$ and $87 \%$, respectively, both increasing with the degree of inflammation (10). Our study showed a comparable diagnostic accuracy, given the limited experience in the interpretation of this tracer and the abundant experience with ${ }^{18}$ F-FDG. However, normal intestinal mucosa has quite variable and sometimes remarkably increased ${ }^{18} \mathrm{~F}$-FDG uptake, rendering the interpretation of ${ }^{18} \mathrm{~F}$-FDG PET imaging more difficult, despite comprehensive experience (25).

To circumvent these disadvantages due to the nonspecific nature of ${ }^{18} \mathrm{~F}$-FDG uptake in the bowel, other scintigraphic techniques that target immune cells have previously been tested for imaging IBD, including polyclonal immunoglobulins and monoclonal antibodies against granulocytes and adhesion molecules $(7,8,26-28)$. Also ${ }^{67} \mathrm{Ga}$-citrate uptake has been shown to correlate with the extent and activity of disease in both Crohn disease and ulcerative colitis $(29,30)$. Autologous leukocytes labeled with either ${ }^{111}$ In-oxine or 99m Tc-hexamethyl-propyleneamine oxime are most used (31). Despite reasonable diagnostic accuracy in targeting IBD lesions, the high cost, the laborious in vitro cell labeling procedure, and the risks associated with handling of potentially contaminated blood have led to relatively limited use of labeled leukocytes as a diagnostic tool in IBD. In this respect, it might be worthwhile to investigate the role of ${ }^{99 m}$ Tc-CXCL8 (quantitative) SPECT/CT in the early identification of active colitis, as neutrophil recruitment is an early and indispensable event in the development of overt colitis.

Imaging tools, such as ${ }^{99 \mathrm{~m}} \mathrm{Tc}-\mathrm{CXCL} 8$, that target specific steps early in disease progression can yield important information on disease characteristics in that particular patient. This might be particularly relevant in efforts to stratify treatment of IBD. Recently, selective CXCR1 and CXCR2 antagonists have been tested in the clinical setting $(32,33)$, and these targeted drugs could be dosed, based on the results of functional imaging studies. CXCL8-based decoy proteins demonstrated strong antiinflammatory activity in vivo in preclinical models (34). Given the results in this study and the translational potential of imaging immune cell recruitment with ${ }^{99 \mathrm{~m}} \mathrm{Tc}-\mathrm{CXCL} 8$ in inflammatory bowel conditions, further prospective studies to validate the tracer specificity and correlation with other noninvasive biomarkers are warranted.

\section{CONCLUSION}

99mTc-CXCL8 scintigraphy is a novel imaging technique that targets intestinal lesions with active colitis with good diagnostic accuracy. This noninvasive technique provides a diagnostic whole-body approach as an alternative to invasive procedures such as endoscopy. Potentially, this tool allows scaling up or stepping down treatment with immune modulating drugs in a personalized fashion. 


\section{DISCLOSURE}

The costs of publication of this article were defrayed in part by the payment of page charges. Therefore, and solely to indicate this fact, this article is hereby marked "advertisement" in accordance with 18 USC section 1734 . This study was funded by internal sources and no external funding was received. No other potential conflict of interest relevant to this article was reported.

\section{REFERENCES}

1. Varol C, Zigmond E, Jung S. Securing the immune tightrope: mononuclear phagocytes in the intestinal lamina propria. Nat Rev Immunol. 2010;10:415-426.

2. Kawalec P, Malinowski KP. Indirect health costs in ulcerative colitis and Crohn's disease: a systematic review and meta-analysis. Expert Rev Pharmacoecon Outcomes Res. 2015;15:253-266.

3. Podolsky DK. Inflammatory bowel disease. N Engl J Med. 2002;347:417-429.

4. Klare P, Poloschek A, Walter B, et al. Single-day sodium picosulfate and magnesium citrat versus split-dose polyethylene glycol for bowel cleansing prior to colonoscopy: a prospective randomized endoscopist-blinded trial. J Gastroenterol Hepatol. 2015;30:1627-1634.

5. Navaneethan U, Kochhar G, Phull H, et al. Severe disease on endoscopy and steroid use increase the risk for bowel perforation during colonoscopy in inflammatory bowel disease patients. J Crohns Colitis. 2012;6:470-475.

6. Rabeneck L, Saskin R, Paszat LF. Onset and clinical course of bleeding and perforation after outpatient colonoscopy: a population-based study. Gastrointest Endosc. 2011;73:520-523.

7. Bruno I, Martelossi S, Geatti O, et al. Antigranulocyte monoclonal antibody immunoscintigraphy in inflammatory bowel disease in children and young adolescents. Acta Paediatr. 2002;91:1050-1055.

8. Bhatti M, Chapman P, Peters M, Haskard D, Hodgson HJ. Visualising E-selectin in the detection and evaluation of inflammatory bowel disease. Gut. 1998;43:40-47.

9. Louis E, Ancion G, Colard A, Spote V, Belaiche J, Hustinx R. Noninvasive assessment of Crohn's disease intestinal lesions with ${ }^{18} \mathrm{~F}-\mathrm{FDG}$ PET/CT. $\mathrm{J}$ Nucl Med. 2007;48:1053-1059.

10. Treglia G, Quartuccio N, Sadeghi R, et al. Diagnostic performance of fluorine18-fluorodeoxyglucose positron emission tomography in patients with chronic inflammatory bowel disease: a systematic review and a meta-analysis. J Crohns Colitis. 2013;7:345-354.

11. Groshar D, Bernstine H, Stern D, et al. PET/CT enterography in Crohn disease: correlation of disease activity on CT enterography with ${ }^{18}$ F-FDG uptake. $\mathrm{J} \mathrm{Nucl}$ Med. 2010;51:1009-1014.

12. Meisner RS, Spier BJ, Einarsson S, et al. Pilot study using PET/CT as a novel, noninvasive assessment of disease activity in inflammatory bowel disease. Inflamm Bowel Dis. 2007;13:993-1000.

13. Stillie R, Farooq SM, Gordon JR, Stadnyk AW. The functional significance behind expressing two IL-8 receptor types on PMN. J Leukoc Biol. 2009;86: $529-543$.

14. Thomas KM, Taylor L, Navarro J. The interleukin- 8 receptor is encoded by a neutrophil-specific cDNA clone, F3R. J Biol Chem. 1991;266:14839-14841.

15. Takata H, Tomiyama H, Fujiwara M, Kobayashi N, Takiguchi M. Cutting edge: expression of chemokine receptor CXCR1 on human effector CD8 $+\mathrm{T}$ cells. $J$ Immunol. 2004;173:2231-2235.

16. Gerdes N, Sukhova GK, Libby P, Reynolds RS, Young JL, Schonbeck U. Expression of interleukin (IL)-18 and functional IL-18 receptor on human vascular endothelial cells, smooth muscle cells, and macrophages: implications for atherogenesis. J Exp Med. 2002;195:245-257.

17. Daig R, Andus T, Aschenbrenner E, Falk W, Scholmerich J, Gross V. Increased interleukin 8 expression in the colon mucosa of patients with inflammatory bowel disease. Gut. 1996;38:216-222.

18. Puleston J, Cooper M, Murch S, et al. A distinct subset of chemokines dominates the mucosal chemokine response in inflammatory bowel disease. Aliment Pharmacol Ther. 2005;21:109-120.

19. Jones SA, Wolf M, Qin S, Mackay CR, Baggiolini M. Different functions for the interleukin 8 receptors (IL-8R) of human neutrophil leukocytes: NADPH oxidase and phospholipase D are activated through IL-8R1 but not IL-8R2. Proc Natl Acad Sci USA. 1996;93:6682-6686.

20. Rennen HJ, Boerman OC, Oyen WJ, Corstens FH. Kinetics of ${ }^{99 \mathrm{~m}} \mathrm{Tc}-$ labeled interleukin-8 in experimental inflammation and infection. J Nucl Med. 2003;44: 1502-1509.

21. Bleeker-Rovers CP, Rennen HJ, Boerman OC, et al. ${ }^{99 \mathrm{~m}} \mathrm{Tc}-$ labeled interleukin 8 for the scintigraphic detection of infection and inflammation: first clinical evaluation. J Nucl Med. 2007;48:337-343.

22. Abrams MJ, Juweid M, tenKate CI, et al. Technetium-99m-human polyclonal $\mathrm{IgG}$ radiolabeled via the hydrazino nicotinamide derivative for imaging focal sites of infection in rats. J Nucl Med. 1990;31:2022-2028.

23. Thaiss CA, Levy M, Suez J, Elinav E. The interplay between the innate immune system and the microbiota. Curr Opin Immunol. 2014;26:41-48.

24. Gerber GK. The dynamic microbiome. FEBS Lett. 2014;588:4131-4139.

25. van Kouwen MC, Nagengast FM, Jansen JB, Oyen WJ, Drenth JP. 2-( $\left.{ }^{18} \mathrm{~F}\right)$ fluoro-2-deoxy-D-glucose positron emission tomography detects clinical relevant adenomas of the colon: a prospective study. J Clin Oncol. 2005;23:37133717.

26. Versaci A, Bonanno N, Baldari S, et al. Diagnostic possibilities and clinical indications of policlonal labeled Ig in the bowel inflammatory disease. Hepatogastroenterology. 1999;46:2260-2264.

27. Alberini JL, Badran A, Freneaux E, et al. Technetium-99m HMPAO-labeled leukocyte imaging compared with endoscopy, ultrasonography, and contrast radiology in children with inflammatory bowel disease. J Pediatr Gastroenterol Nutr. 2001;32:278-286.

28. Lachter J, Isseroff HN, Yasin K, Keidar Z, Israel O. Radiolabeled leukocyte imaging in inflammatory bowel disease: a prospective blinded evaluation. Hepatogastroenterology. 2003;50:1439-1441.

29. Jones B, Abbruzzese AA, Hill TC, Adelstein SJ. Gallium-67-citrate scintigraphy in ulcerative colitis. Gastrointest Radiol. 1980;5:267-272.

30. Rhodes RW, Bianco JA, Anderson ML, Shafer RB. Scintigraphic observations using gallium-67 citrate in a patient with Crohn's disease. Minn Med. 1981;64: $469-471$.

31. Sans M, Fuster D, Llach J, et al. Optimization of technetium-99m-HMPAO leukocyte scintigraphy in evaluation of active inflammatory bowel disease. Dig Dis Sci. 2000;45:1828-1835.

32. Lazaar AL, Sweeney LE, MacDonald AJ, Alexis NE, Chen C, Tal-Singer R. SB656933, a novel CXCR2 selective antagonist, inhibits ex vivo neutrophil activation and ozone-induced airway inflammation in humans. Br J Clin Pharmacol. 2011;72:282-293.

33. Chapman RW, Phillips JE, Hipkin RW, Curran AK, Lundell D, Fine JS. CXCR2 antagonists for the treatment of pulmonary disease. Pharmacol Ther. 2009;121: 55-68.

34. Falsone A, Wabitsch V, Geretti E, et al. Designing CXCL8-based decoy proteins with strong anti-inflammatory activity in vivo. Biosci Rep. 2013;33:743-754. 